\title{
Direct and indirect anti-leukemic properties of activity-on-target interferons for the treatment of T-cell acute lymphoblastic leukemia
}

T-cell acute lymphoblastic leukemias (T-ALL) are rare aggressive hematologic tumors resulting from the malignant transformation of T-cell progenitors in the thymus. ${ }^{1} \mathrm{~T}$-ALL treatment currently consists of high-dose multi-agent chemotherapy, potentially followed by hematopoietic stem cell transplantation for high-risk patients. ${ }^{2}$ Unfortunately, even with such a harsh and long treatment regime, the outcome of T-ALL patients with primary refractory or early relapsed leukemia remains poor. Novel therapeutic strategies to treat those high-risk patients remain an important unmet medical need. ${ }^{3}$ Type-I interferons (IFN-I) have a long history in the treatment of cancer, including hematologic malignancies. ${ }^{4}$ The pleiotropic anticancer effects induced by IFN-I result from a combination of: (i) direct cancer cell growth inhibition by inducing cell cycle arrest, apoptosis, and differentiation; and (ii) indirect effects by the activation of the immune system involving antigen presentation by dendritic cells and priming of cytotoxic $\mathrm{CD} 8^{+} \mathrm{T}$ cells. ${ }^{5}$ As such, this cytokine, with both direct and indirect immunostimulatory anti-cancer effects, is unique in its kind. Nevertheless, IFN-I therapy experienced variable and unpredictable success in the clinic. Its clinical application is severely impeded by a complex pattern of adverse side-effects, due to the multifaceted pattern of activity of IFN-I. ${ }^{6}$ Therefore, safe exploitation of the anti-cancer potential of IFN-I requires strategies to direct its activity to selected target cells only.

To document the direct anti-cancer effect of IFN-I on T-ALL, we stimulated nine human T-ALL cell lines with increasing concentrations of recombinant human interferon alpha-2 (hIFNa2) for 4 days and measured the effect on their in vitro growth. A variable anti-proliferative effect was observed, with no correlation between sensitivity to IFN-I and immunophenotype or molecular subtype (Online Supplementary Figure S1). Consistent with previous studies, ${ }^{7,8}$ the anti-proliferative effect was associated with activation of the JAK/STAT1 pathway as documented by the increased levels of phosphorylated STAT1 (pSTAT1) determined by flow cytometry. We hypothesized that elevated PSTAT1 levels upon IFN-I stimulation could be used as a biomarker to stratify between IFN-I-sensitive and non-sensitive T-ALL patients. To validate our findings in primary T-ALL patients' material, we stimulated three patient-derived xenografts (PDX) samples with $100 \mathrm{ng} / \mathrm{mL}$ hIFN 22 for 30 min and quantified pSTAT1 levels (Figure 1A). As for the cell lines, a variable response to IFN-I was observed. Subsequently, we transplanted the IFN-I-responsive (PDX\#1) and non-responsive (PDX\#3) PDX samples into immunodeficient NOD-scid IL2Rgnull (NSG) mice. As soon as evidence of leukemia progression was observed ( $>5 \%$ human $\mathrm{CD}_{4} 5^{+}\left[\mathrm{hCD} 45^{+}\right]$cells in peripheral blood), mice (5 mice/group) were treated for 7 consecutive days with intraperitoneal injections of $30 \mathrm{mg} \mathrm{hIFN \alpha 2/mouse} \mathrm{or}$ vehicle (100 $\mu \mathrm{L}$ phosphate-buffered saline). A significant anti-leukemic effect was observed only for the IFN-I-responsive PDX\#1, with a decrease in the percentage of hCD $45^{+}$cells in the peripheral blood at the end of the 7-day treatment regime and a prolonged leukemia-free survival of the mice (Figure 1B). Of note, IFN $\alpha 2$ has strict species specificity and human IFNa2 is unable to activate the mouse receptor complex, ${ }^{7,9}$ indicating that the observed anti-leukemic effects can only be due to cell-intrinsic direct effects on the human leukemia cells.

To further document the indirect immune-mediated antileukemic properties of IFNa2, we generated an experimental T-ALL model that could be exploited to monitor leukemia progression both in an immunocompetent background as well as an immunodeficient background. To do this, we intercrossed the $L c k-c r e^{t g /+} ; P t e n^{f l / f l}$ spontaneous murine T-ALL model ${ }^{10}$ with a ROSA26-eGFP/luciferase reporter line ${ }^{11}$ on a pure C57BL/6 background and derived eight primary murine T-ALL cell lines from diseased mice (Online Supplementary Figure S1C). As for the human TALL cell lines, a variable anti-proliferative effect with increased percentages of apoptotic cells was observed when murine T-ALL were treated for 3 days in vitro with increasing concentrations of recombinant mouse interferon alpha-2 (mIFNa2) (Online Supplementary Figure S1D, $E)$. Subsequently, we transplanted the murine T-ALL cell lines in both immunodeficient NSG as well as immunocompetent syngeneic mice and treated them with intraperitoneal injections of $30 \mu \mathrm{g} \mathrm{mIFN} \alpha 2 /$ mice or vehicle for 7 consecutive days (Figure 1C). As the injected T-ALL lymphoblasts express the eGFP-Firefly luciferase reporter from the Rosa26 promoter, leukemic burden could be efficiently monitored using in vivo bioluminescence imaging." mIFNa2 treatment resulted in a significant reduction of the leukemic burden, and prolonged survival of both immunodeficient and immunocompetent mice transplanted with murine T-ALL.

Notably, the therapeutic efficacy of mIFNa2 was consistently higher in the presence of an intact immune system (Figure 1C), suggesting an additive immune-mediated antileukemic effect. To uncouple these direct and putative indirect anti-leukemic properties of mIFNa2, we used our recently developed cell-type specific Activity-on-Target 
A

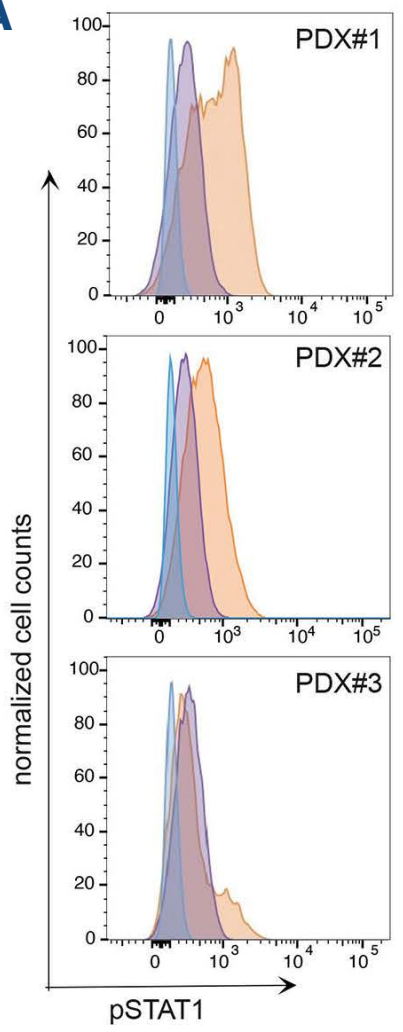

unstained, unstimulated

stained, unstimulated

stained, hIFNa2 stimulated $100 \mathrm{ng} / \mathrm{ml} 30 \mathrm{~min}$
PDX\#1 (hIFNo2 responsive)

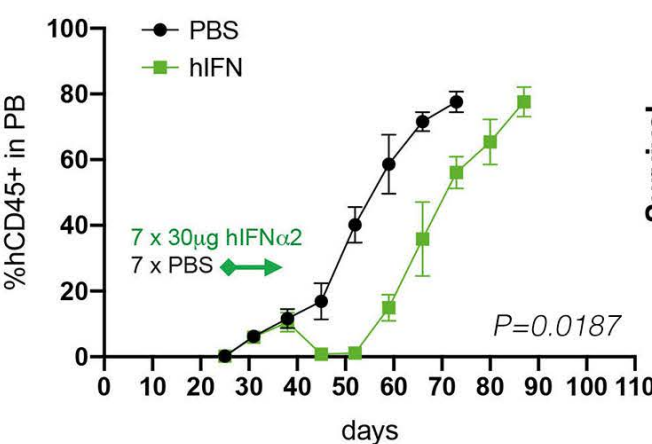

days

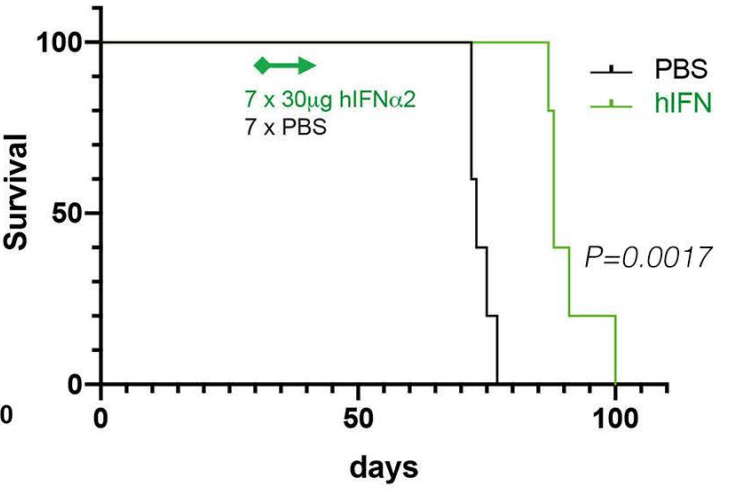

PDX\#3 (hIFN 22 non-responsive)
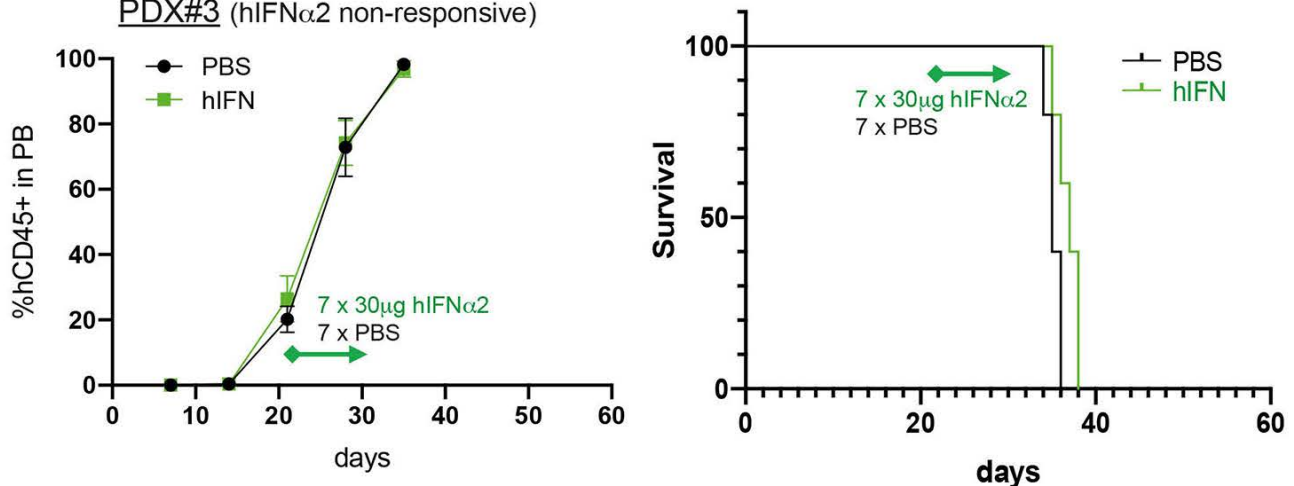

C murine T-ALL cells

HOST: NSG mice
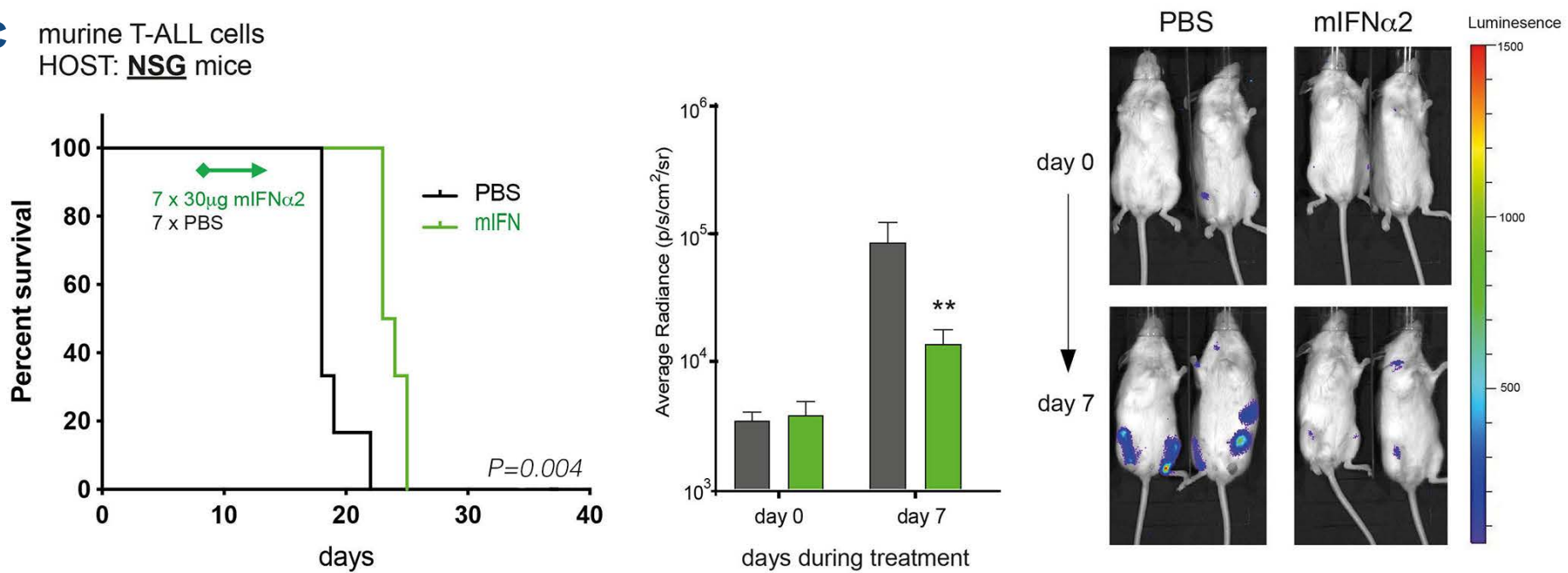

murine T-ALL cells

HOST: $\underline{\text { C57BL/6 }}$ mice
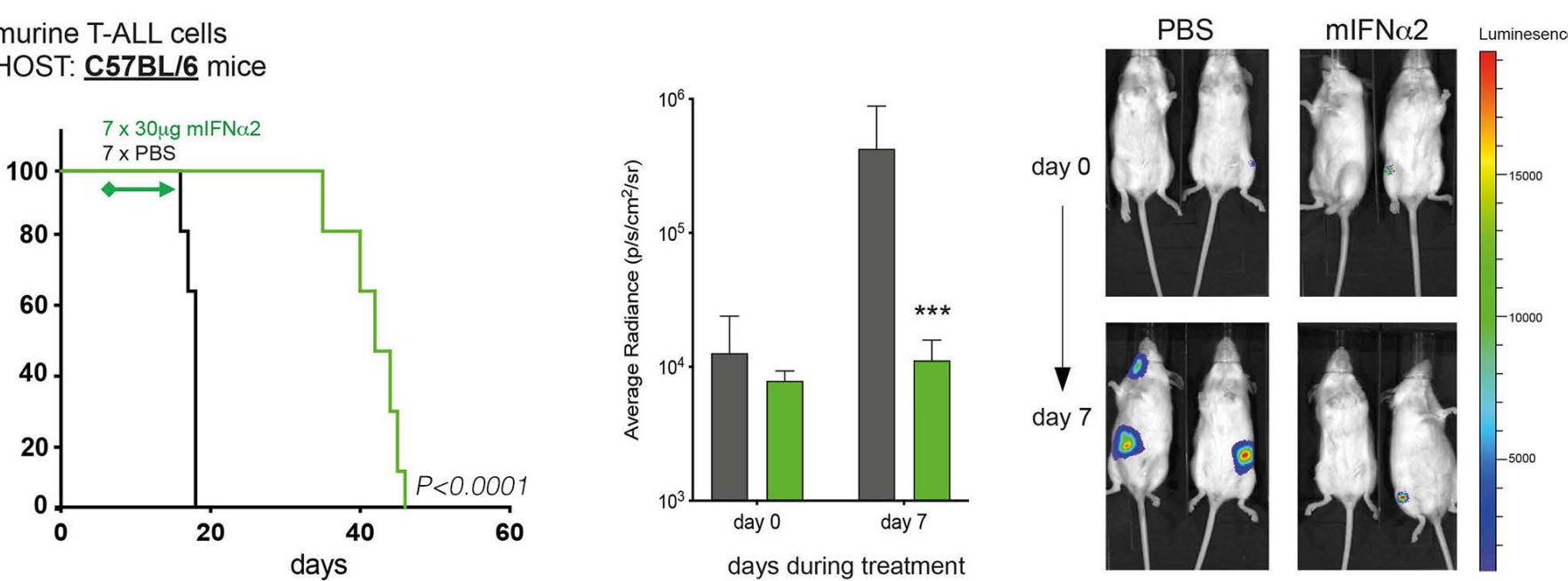

Figure 1. Anti-leukemic effects of interferon alpha-2 on the progression of human and murine T-cell acute lymphoblastic leukemia in vivo. (A) Flow cytometric analysis of phospho-STAT1 (pSTAT1) levels in three established T-cell acute lymphoblastic leukemia (T-ALL) patient-derived xenografts (PDX) after $30 \mathrm{~min}$. ex vivo stimulation with 100 ng recombinant human interferon

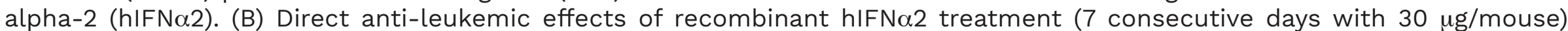
versus vehicle on type I interferon-responsive (PDX\#1) and non-responsive (PDX\#3) T-ALL patient-derived xenograft model transplanted in NSG mice (5 mice/group). Effect on leukemia progression was measured by flow cytometric quantification of the percentage of hCD $45^{+}$cells in peripheral blood (left) and Kaplan-Meier survival curves (right). (C) Anti-leukemic effects of recombinant murine interferon alpha-2 (mIFNa2) treatment ( 7 consecutive days; 30 mg/mouse) versus vehicle control (phosphate-buffered saline, PBS) on in vivo progression of murine T-ALL cell line transplanted in immunodeficient NSG animals or immunocompetent syngeneic C57BL/6 animals. Leukemia burden was quantified via in vivo bioluminescence imaging during treatment (right) and by Kaplan-Meier survival curves (left). 
interferons (AcTaferons; AFN).,72-15 To obtain these AFN, we fused the hIFNa2 ${ }^{\mathrm{Q} 124 \mathrm{R}}$, a human IFN $\alpha 2$ mutation that enables binding to the murine interferon receptor complex with a 100-fold reduced affinity compared to wild-type (WT) mIFN 2 2, to $\mathrm{VHH}$ single domain antibodies targeting the murine CD8 (mCD8-AFN). We specifically chose the
mCD8-AFN, as this CD8a epitope is not only present on $40 \%$ of T-ALL cases, but also on mouse classical dendritic cell type 1 (CDC1) which are particularly important for eliciting a $\mathrm{CD}^{+}$cytotoxic $T$ lymphocyte response to kill cancerous cells upon IFN $\alpha 2$ stimulation. ${ }^{12}$ Evaluating the therapeutic effects of the mCD8-AFN on both $\mathrm{CD}^{+}$versus
A

T-ALL cells: CD8-

HOST: NSG mice

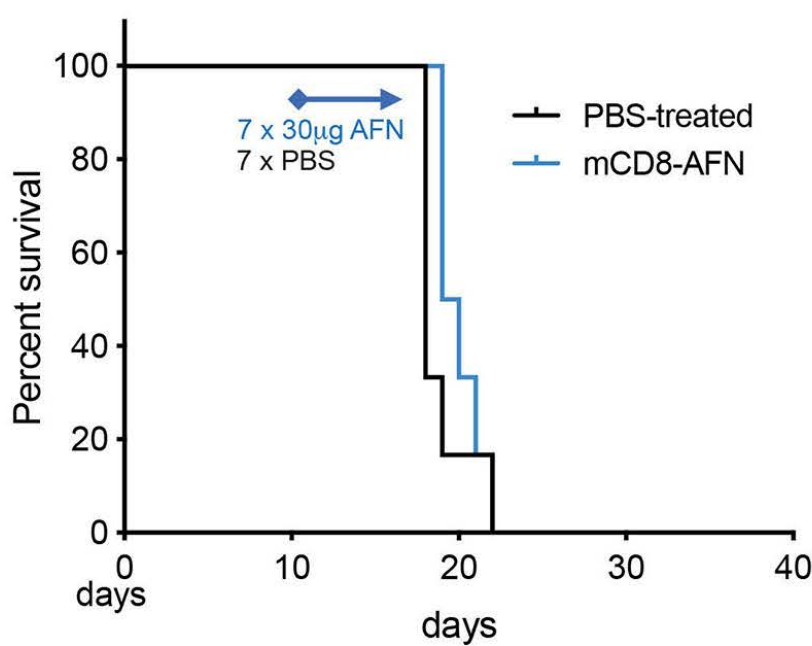

T-ALL cells: CD8+ HOST: NSG mice

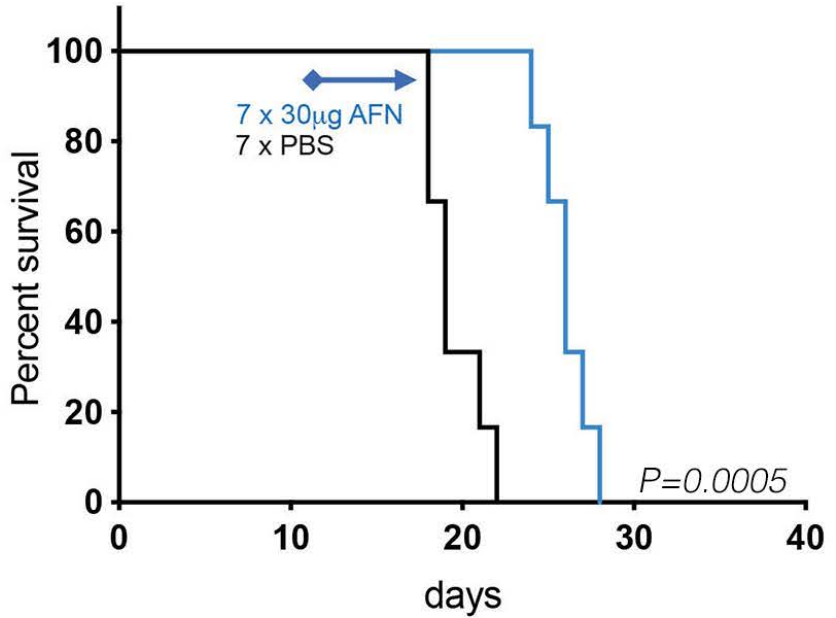

$\mathbf{B}$

T-ALL cells: CD8HOST: C57BL/6 mice

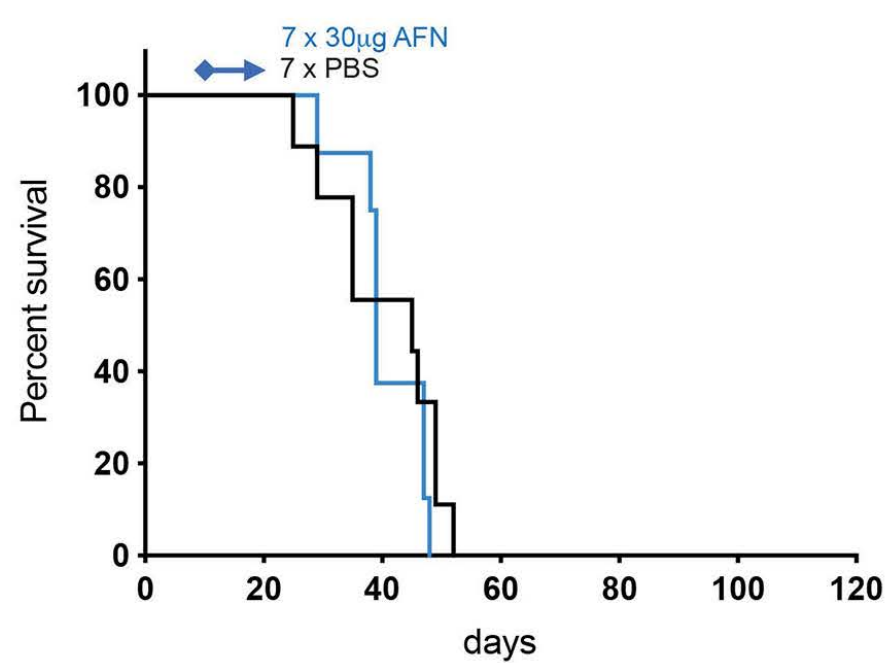

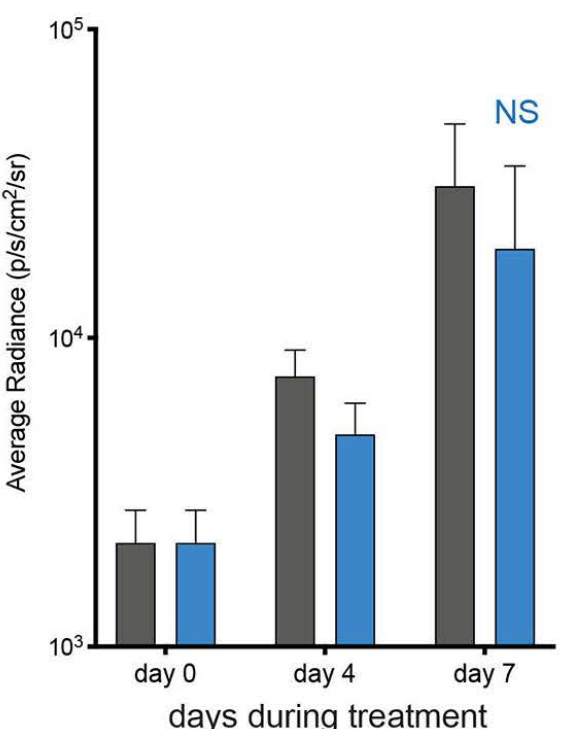
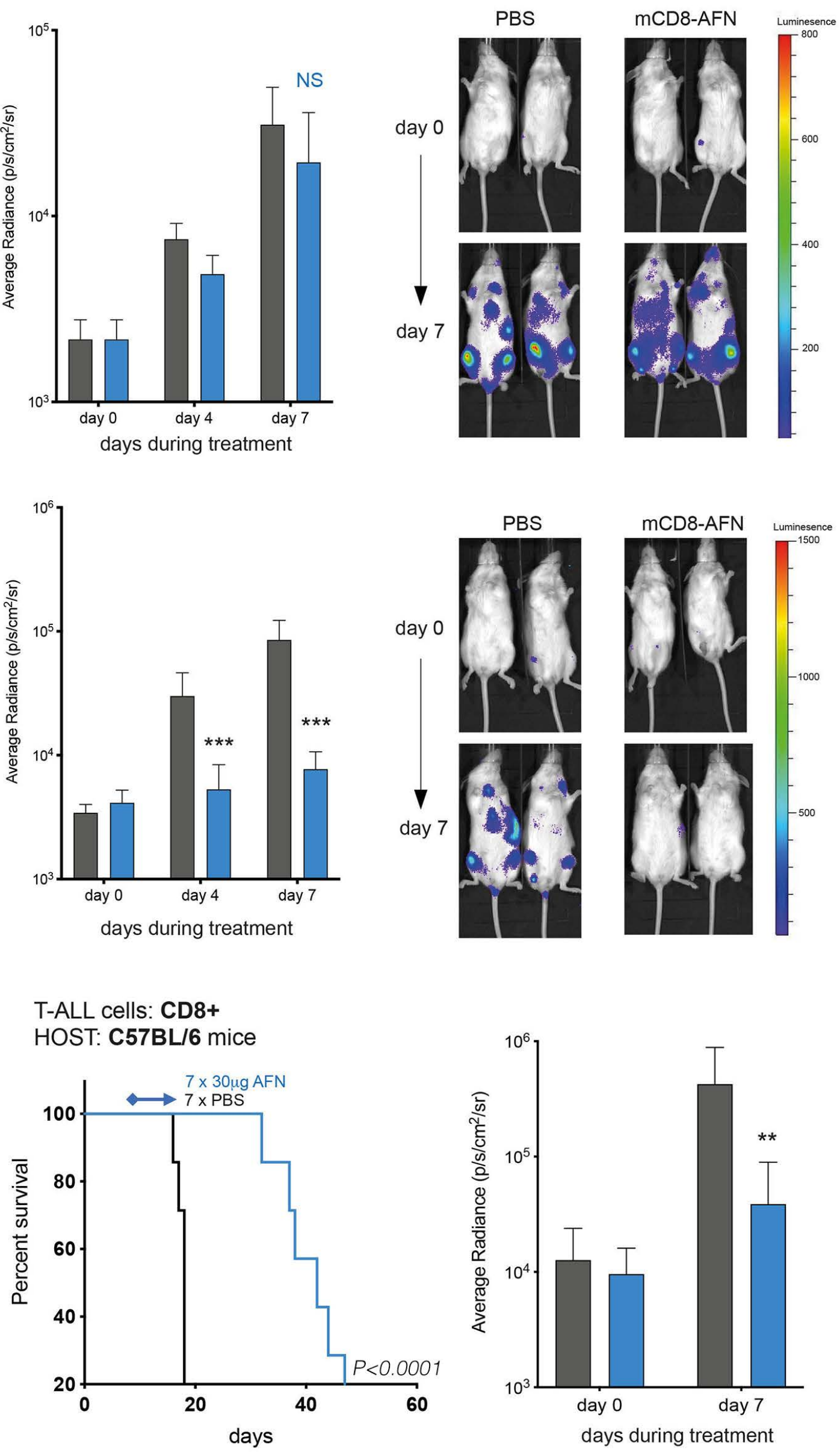

Figure 2. mCD8-AFN has direct but no indirect immune-mediated anti-leukemic effects on murine T-cell acute lymphoblastic leukemia. (A) Anti-leukemic effect of mCD8-AFN treatment ( 7 consecutive days; $30 \mu g / m o u s e)$ versus vehicle (phosphatebuffered saline, PBS) on in vivo progression of $\mathrm{CD}^{-}$and $\mathrm{CD} 8^{+}$murine $\mathrm{T}$-cell acute lymphoblastic leukemia (T-ALL) cell lines transplanted in immunodeficient NSG mice or (B) in syngeneic C57BL/6 mice. Leukemic burden was quantified via in vivo bioluminescence imaging during treatment regime (right) and Kaplan-Meier survival curves (left). 
A

T-ALL cells: CD8-

HOST: NSG mice

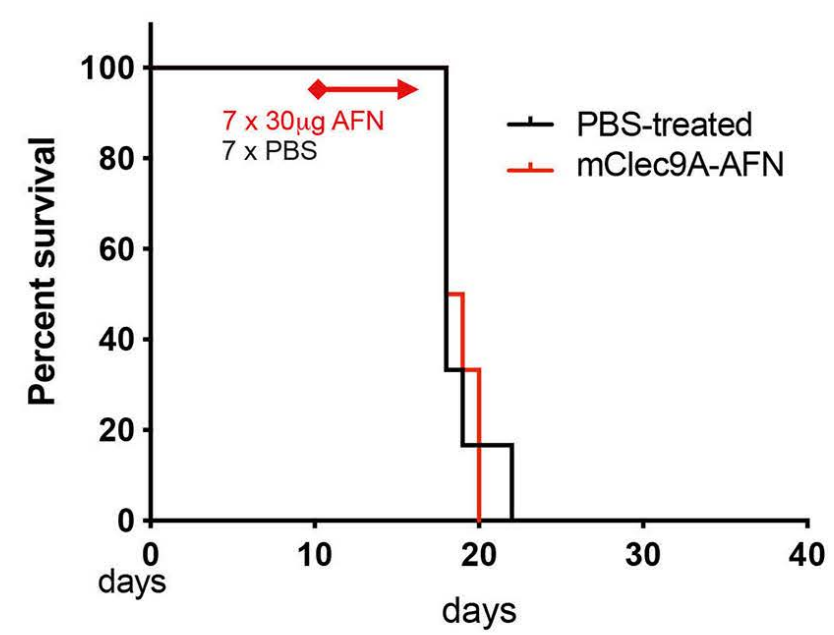

B

T-ALL cells: CD8HOST: C57BL/6 mice

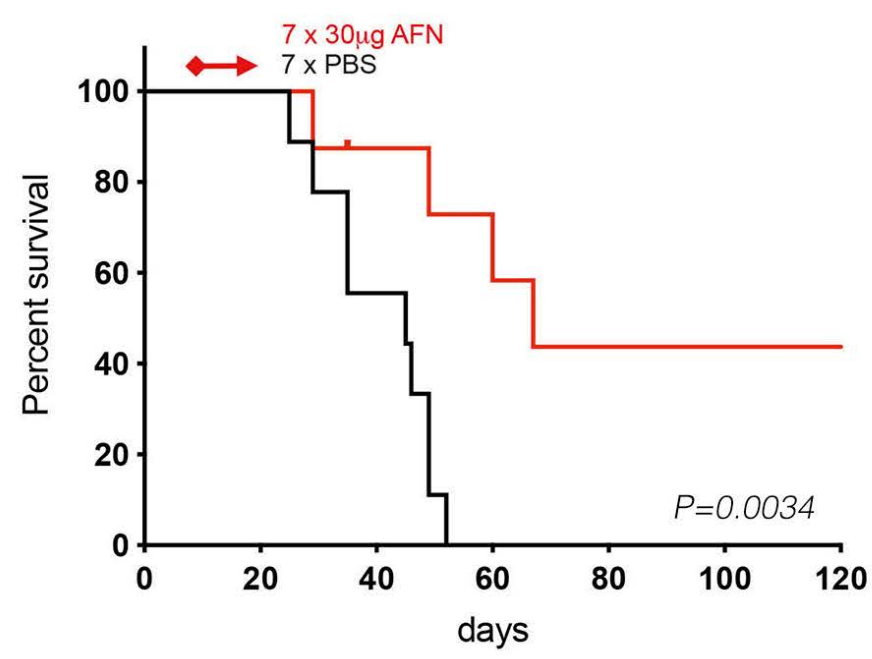

T-ALL cells: CD8+ HOST: NSG mice

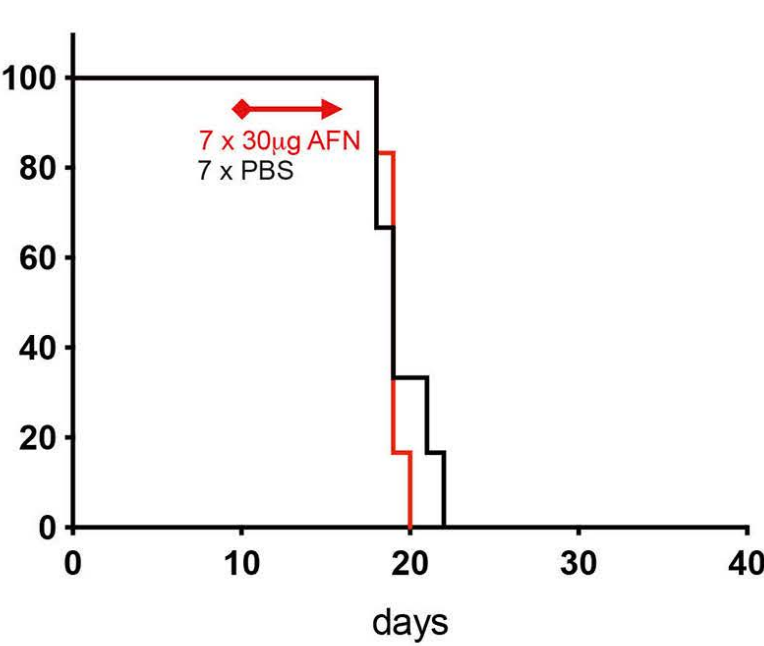

T-ALL cells: CD8+ HOST: C57BL/6 mice

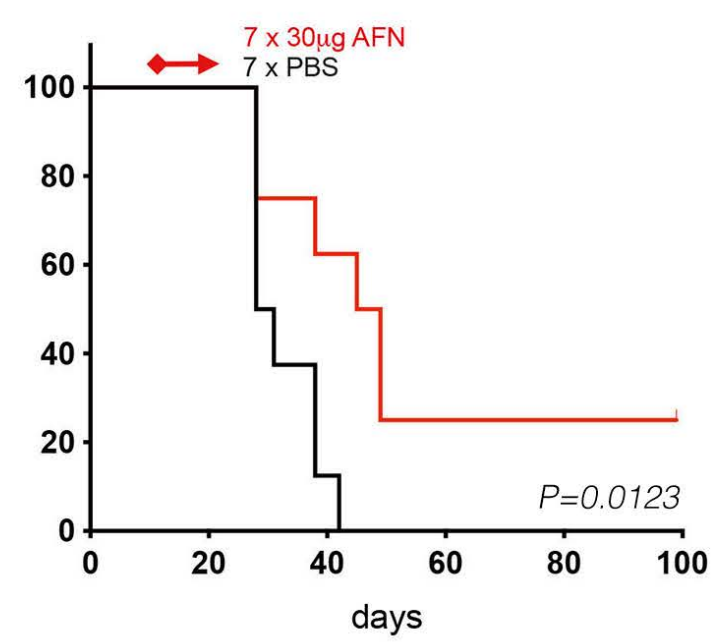

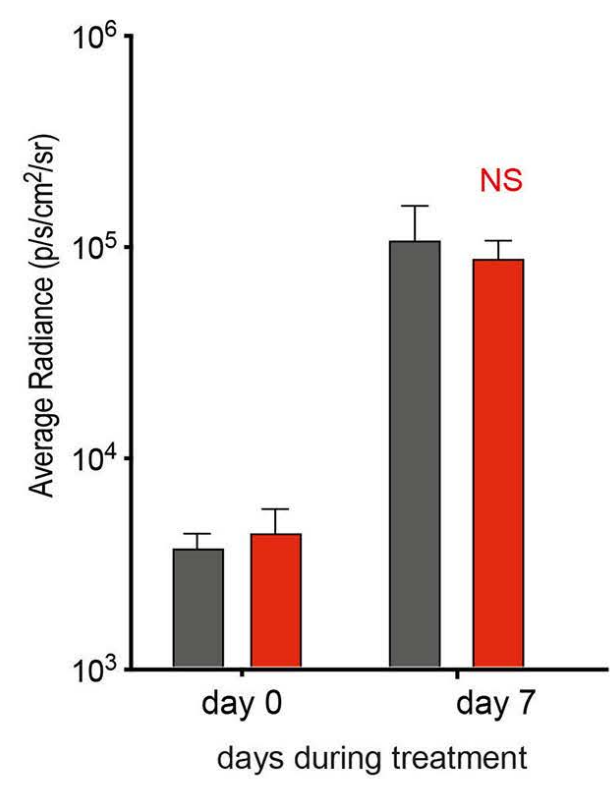

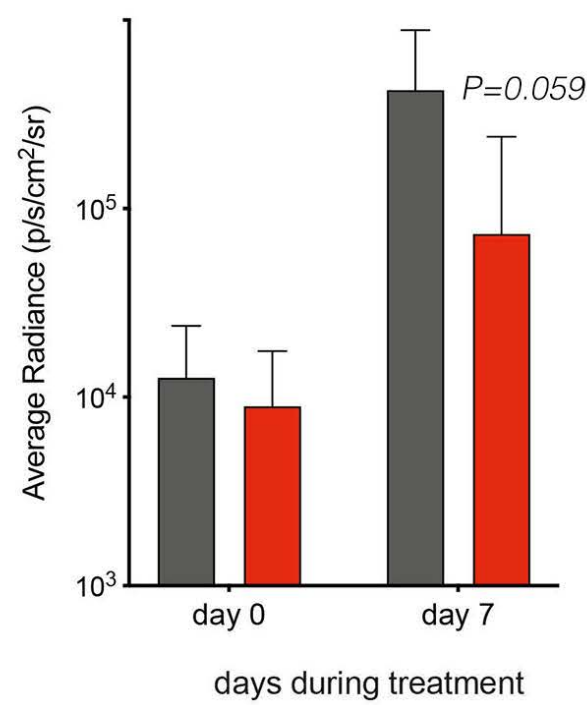

Figure 3. mClec9A-AFN has indirect but no direct anti-leukemic effects on murine T-cell acute lymphoblastic leukemia. (A) Anti-leukemic effect of mClec9A-AFN treatment ( 7 consecutive days; $30 \mathrm{mg} / \mathrm{mouse}$ ) versus vehicle (phosphate-buffered saline, $\mathrm{PBS}$ ) on in vivo progression of $\mathrm{CD}^{-}$and $\mathrm{CD}^{+}$murine T-cell acute lymphoblastic leukemia (T-ALL) cell lines transplanted in immunodeficient NSG mice or (B) in syngeneic C57BL/6 mice. Leukemic burden was quantified via in vivo bioluminescence imaging during treatment regime (right) and Kaplan-Meier survival curves (left).

CD8- T-ALL in an immunocompetent versus immunodeficient background, allowed us to dissect the roles of the direct versus indirect anti-leukemic actions of IFN 2 2. To compare our results to those of a WT IFN 2 with a similar molecular weight as the AFN, we coupled this cytokine to a single-domain antibody targeting Bcll10, an epitope that is absent in mice (mIFNa2-WT). As expected, the mCD8AFN had only an anti-proliferative effect on the $C D 8^{+} \mathrm{T}-$ ALL and not on the CD8- T-ALL cell lines in vitro (Online Supplementary Figure $S 2 A-C$ ), confirming its cell-specificity. To evaluate the cell-specific direct anti-leukemic properties of mCD8-AFN on the growth of murine CD8 ${ }^{+}$ $\mathrm{T}-\mathrm{ALL}$ in an in vivo context, we transplanted both $\mathrm{CD} 8^{+}$and CD8- T-ALL cell lines into immunodeficient NSG mice. As expected, the $\mathrm{mCD} 8-\mathrm{AFN}$ had a significant direct anti-leukemic effect only on the CD8 $8^{+}$T-ALL cell line, with a significant decrease of bioluminescence during the treatment and prolonged survival (Figure $2 \mathrm{~A}$ ). Of note, the direct antileukemic effect of the mCD8-AFN was more efficient than that of WT mIFNa2 (Online Supplementary Figure S3). As
AFN do not efficiently bind the ubiquitously expressed interferon receptor complex, they will not be cleared from the circulation before reaching their desired target population. This so-called 'sink-effect' can explain the improved direct anti-leukemic effect seen with the AFN.

As the CD8a epitope of the mCD8-AFN is also present on cDC1 cells, which have been shown to be superior in antigen cross-presentation, and on effector $C D 8^{+} T$ cells necessary to kill tumor cells, we hypothesized that the mCD8-AFN would also be able to induce an indirect antileukemic response. To test this, we repeated the experiment in an immunocompetent background. As an indirect immune-mediated effect would be independent of the immunophenotype of the T-ALL cells, we hypothesized that, in this setting, we would observe an anti-leukemic effect of the mCD8-AFN on both CD8 $8^{-}$and CD8 ${ }^{+}$T-ALL. However, no effect could be seen on the CD8- T-ALL, only on the CD8 ${ }^{+}$ T-ALL (Figure 2B), indicating that mCD8-AFN has only direct anti-leukemic T-ALL properties, and is not able to induce an indirect immune-mediated anti-leukemic response. 
The inability of mCD8-AFN to elicit an immune-mediated anti-leukemic response could be either due to mature T-cell aplasia and leukemia progression in these mice, or due to the intrinsic nature of the AFN. We therefore tested the anti-leukemic properties of a second AFN, mClec9a-AFN, which we previously used to efficiently induce a cDC1-mediated anti-tumor response in the context of melanoma, breast carcinoma and lymphoma..$^{12}$ As Clec9A is not expressed in normal or malignant $T$ cells, no direct anti-leukemic properties could be observed in vitro (Online Supplementary Figure S2A-C) or in vivo in immunodeficient NSG mice (Figure $3 \mathrm{~A}$ ). To evaluate the indirect immune-mediated anti-leukemic effects of mClec9A-AFN, we repeated the experiment in syngeneic C57BL/ 6 mice ( 7 mice/group). This time, a significant indirect effect on leukemic progression could be seen in the transplanted mice, irrespective of whether they were transplanted with $\mathrm{CD}^{+}$or $\mathrm{CD} 8^{-} \mathrm{T}-\mathrm{ALL}$ (Figure 3B). These data demonstrate that mClec $9 \mathrm{~A}-\mathrm{AFN}$ is able to induce a strong immune-mediated anti-tumor response. However, we currently cannot explain why we saw a difference between the ability of mCD8-AFN and mClec9A-AFN to induce an indirect immune-mediated anti-leukemic effect. In the mouse, the Clec9A-epitope is also present on CD8-immune cell subtypes, which may contribute to the immune response. Alternatively, binding of the CD8AFN on cytotoxic CD8 ${ }^{+} \mathrm{T}$ cells may (partially) hamper their cytotoxic functions, although the anti-CD8 singledomain antibody that was used for the design of the mCD8-AFN was demonstrated to be a non-neutralizing antibody. ${ }^{15}$

Finally, the adverse side-effects (body weight loss and hematologic deficiencies) observed in mice treated with mIFN $\alpha 2-W T$ were significantly reduced in mice treated with the AcTaferons (Online Supplementary Figure S2D, E), as shown before. ${ }^{7,12,13}$

In summary, we used preclinical mouse models of T-ALL to show that immunocytokines with cell-specific activity can preserve the anti-leukemic properties of IFN $\alpha 2$ with a concomitant reduction of systemic toxic side-effects. As such, these AcTaferons can be used as a direct anti-leukemic agent in combination with classical chemotherapy, or as off-the-shelf targeted immunotherapy for $\mathrm{T}$-cell malignancies.

\section{Authors}

Steven Goossens,,${ }^{1,2,3, *}$ Anje Cauwels, ${ }^{1,3,4,5, *}$ Tim Pieters, ${ }^{1,3}$ Renate De
Smedt, ${ }^{1,3}$ Sara T'Sas, ${ }^{1,3}$ André Almeida, ${ }^{1,3}$ Willem Daneels, ${ }^{1,6}$ Pieter Van Vlierberghe ${ }^{1,3, \#}$ and Jan Tavernier ${ }^{1,3,4,5, \#}$

${ }^{1}$ Cancer Research Institute Ghent (CRIG), Ghent University;

${ }^{2}$ Department of Diagnostic Sciences, Ghent University; ${ }^{3}$ Department of Biomolecular Medicine, Ghent University; ${ }^{4} \mathrm{VIB}-\mathrm{UGent}$ Center for Medical Biotechnology; ${ }^{5}$ Orionis Biosciences BV and ${ }^{6}$ Department of Hematology, Ghent University Hospital, Ghent, Belgium

*SG and AC contributed equally as co-first authors.

"PVV and JT contributed equally as co-last authors.

Correspondence:

PIETER VAN VLIERBERGHE - pieter.vanvlierberghe@ugent.be https://doi.org/10.3324/haematol.2021.278913

Received: April 5, 2021.

Accepted: July 2, 2021.

Prepublished: October 14, 2021.

\section{Disclosures}

JT and AC are affiliated with Orionis Biosciences. JT holds equity interests and receives financial research support from Orionis Biosciences. The remaining authors declare that the research was conducted in the absence of any commercial or financial relationships that could be construed as a potential conflict of interest.

\section{Contributions}

SG, AC, PVV and JT conceived and designed the experiments and wrote the paper. SG, AJ, TP, ST and RDS performed experiments. SG and ST analyzed data. SG, TP and AA generated the mouse T-cell acute lymphoblastic leukemia cell lines. AC and JT produced the cytokines. SG, AC, WD, PVV and JT discussed results and critically reviewed the manuscript. SG and PVV directed and supervised the research. All authors approved the final manuscript.

\section{Acknowledgments}

We thank Reza Hassanzadeh Ghassabeh (VIB Nanobody Core) for the selection of the VHH. JT is a recipient of UGent Methusalem and ERC Advanced (CYRE, n. 340941) grants.

\section{Funding}

This work was supported by the Stichting ME TO YOU, the Ghent University Research Fund (BOF-UGent) and the Research Foundation Flanders (FWO).

\section{Data-sharing statement}

Original data can be made available in response to a reasonable, written request to the corresponding author.

\section{References}

1. Meijerink JP. Genetic rearrangements in relation to immunophenotype and outcome in T-cell acute lymphoblastic leukaemia. Best Pract Res Clin Haematol. 2019;23(3):307-318.
2. Hofmans M, Suciu S, Ferster A, et al. Results of successive EORTCCLG 58881 and 58951 trials in paediatric T-cell acute lymphoblastic leukaemia (ALL). Br J Haematol. 2019;186(5):741-753. 
3. Hunger SP, Mullighan CG. Acute lymphoblastic leukemia in children. N Engl J Med. 2015;373(16):1541-1552.

4. Parker BS, Rautela J, Hertzog PJ. Antitumour actions of interferons: implications for cancer therapy. Nat Rev Cancer. 2016;16(3):131-144.

5. Demerdash Y, Kain B, Essers MAG, King KY. Yin and yang: the dual effects of interferons on hematopoieisis. Exp Hematol. 2021;96:1-12.

6. Jonasch E, Haluska FG. Interferon in oncological practice: review of interferon biology, clinical applications, and toxicities. Oncologist. 2001;6(1):34-55.

7. Garcin G, Paul F, Staufenbiel M, et al. High efficiency cellspecific targeting of cytokine activity. Nat Commun. 2014;5:3016.

8. Lesinski GB, Anghelina M, Zimmerer J, et al. The antitumor effects of IFN-alpha are abrogated in a STAT1-deficient mouse. J Clin Invest. 2003;112(2):170-180.

9. Weber H, Valenzuela D, Lujber G, Gubler M, Weissmann C. Single amino acid changes that render human IFN- $\alpha 2$ biologically active on mouse cells. EMBO J. 1987;6(3):591-598.
10. Suzuki A, Yamaguchi MT, Ohteki T, et al. T cell-specific loss of Pten leads to defects in central and peripheral tolerance. Immunity. 2001;14(5):523-534.

11. Pieters T, T'Sas S, Demoen L, et al. Novel strategy for rapid functional in vivo validation of oncogenic drivers in haematological malignancies. Sci Rep. 2019;9(1):10577.

12. Cauwels A, Van Lint S, Paul F, et al. Delivering type I interferon to dendritic cells empowers tumor eradication and immune combination treatments. Cancer Res. 2018;78(2):463-474.

13. Cauwels A, Van Lint S, Garcin G, et al. A safe and highly efficient tumor-targeted type I interferon immunotherapy depends on the tumor microenvironment. Oncoimmunology. 2017;7(3):e1398876.

14. Cauwels A, Van Lint S, Catteeuw D, et al. Targeting interferon activity to dendritic cells enables in vivo tolerization and protection against EAE in mice. J Autoimmun. 2019;97:70-76

15. Huyghe L, Van Parys A, Cauwels A, et al. Safe eradication of large established tumors using neovasculature-targeted tumor necrosis factor-based therapies. EMBO Mol Med.

2020;12(2):e11223. 